\author{
Maestría en Economía \\ Facultad de Ciencias Económicas \\ Universidad Nacional de La Plata
}

TESIS DE MAESTRIA

ALUMNO

Federico Cerimedo

TITULO

Duración del Desempleo y Ciclo Económico en la Argentina

\author{
DIRECTOR \\ Leonardo Gasparini \\ FECHA DE DEFENSA \\ $4 / 20 / 2004$
}




\title{
Duración del Desempleo y Ciclo Económico en la Argentina
}

\author{
Federico Cerimedo*
}

29 de marzo de 2004

\begin{abstract}
Resumen
El estado del ciclo económico ha tenido incidencia sobre la duración del desempleo en la Argentina. En este trabajo se ofrecen distintas alternativas para cuantificar este efecto. Se estima un modelo para la probabilidad de experimentar un tránsito de la desocupación al empleo, a partir de historias de desempleo individuales construidas con el panel rotatorio de la Encuesta Permanente de Hogares. La muestra cubre el área del Gran Buenos Aires durante el período 1995-2003. Entre los resultados obtenidos, se encuentra que cuando la economía crece $4 \%$, la probabilidad de experimentar un tránsito del desempleo al empleo es 16,2\% mayor que cuando se contrae a igual tasa. A su vez, se comprueba que este efecto no es homogéneo entre sectores. Se halla que la tasa de salida del desempleo es $31 \%$ mayor para las personas con último empleo en el sector industrial respecto a aquellas que trabajaron por última vez en el sector servicios.
\end{abstract}

Palabras clave: desempleo, empleo, duración, ciclo económico, Argentina.

Clasificación JEL: J6, E32.

\footnotetext{
* Tesis de la Maestría en Economía de la Facultad de Ciencias Económicas de la Universidad Nacional de La Plata. Agradezco los valiosos comentarios de Leonardo Gasparini, director de esta tesis. Todos los errores son de mi responsabilidad. Los comentarios son bienvenidos en fcerimedo@infovia.com.ar.

Una versión de este trabajo concursó en el Premio "Profesor Elías Salama” en el ámbito de las Novenas Jornadas de Economía Monetaria e Internacional, organizado por el Departamento de Economía de la Universidad Nacional de La Plata, en el cual obtuvo el primer premio.
} 


\section{Indice}

1. Introducción...................................................................................................

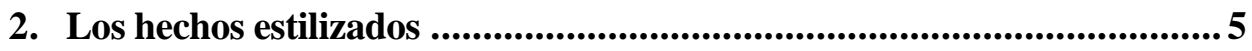

2.1. Flujos hacia el desempleo ...................................................... 6

2.2. Duración de los episodios de desempleo ....................................... 9

3. El modelo econométrico.................................................................... 13

4. La construcción de los datos ..................................................................14

5. Los resultados econométricos.......................................................... 19

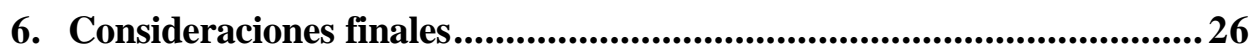

7. Referencias Bibliográficas...............................................................227 


\section{Introducción}

El desempleo ha sido una de las dificultades más importantes que ha enfrentado la economía argentina desde el comienzo de la década del 90. A pesar de que el país logró estabilidad nominal y elevadas tasas de crecimiento del producto, la tasa de desocupación se incrementó de manera considerable. Desde valores cercanos al $5 \%$ en la década del 80 pasó a niveles ostensiblemente más elevados en la década del 90 y los años siguientes, con un pico algo superior al 20\% en mayo de 1995 y otro que alcanzó el 22\% en el mismo mes de 2002 .

Inicialmente, el aumento del desempleo estuvo asociado a la elevada tasa de destrucción de empleos que naturalmente trajo aparejado el proceso de reestructuración económica que tuvo lugar con las reformas implementadas (apertura comercial y financiera, desregulación y privatizaciones). Con una economía en crecimiento, la tasa de creación de empleo también era relativamente elevada, pero no lo suficiente como para evitar el aumento del desempleo. En ese marco, la duración del desempleo era relativamente baja.

El crecimiento económico de la primera mitad de la década del 90 estuvo alimentado por el ingreso de capitales, primero en forma de privatizaciones y otras inversiones en el sector real de la economía, y luego en forma de endeudamiento. ${ }^{1}$ Tras los sucesivos shocks macroeconómicos externos (crisis del Tequila, crisis asiática con devaluaciones competitivas, caída de términos de intercambio, fortalecimiento del dólar, devaluación de Brasil) el ingreso de capitales mermó y el nivel de actividad económica se resintió progresivamente, hasta reducirse de manera brusca durante 2001 e inicios de 2002, con la caída del esquema de Convertibilidad. En este contexto, los problemas de desocupación también se intensificaron.

Dado que la tasa de desocupación depende tanto de las entradas al desempleo como de las salidas del mismo, analizar la duración del desempleo no es en general suficiente para obtener conclusiones acerca del nivel de aquella tasa. Sin embargo, es altamente probable que, en el contexto recesivo imperante a partir de la segunda mitad de la década del 90, la

\footnotetext{
${ }^{1}$ Ver por ejemplo Heymann (2000).
} 
duración del desempleo se haya incrementado, por lo que resulta interesante estudiar qué ocurrió con las tasas de salida del desempleo en ese período.

Varios trabajos empíricos abordan diferentes aspectos de los fenómenos de duración en el mercado laboral de la Argentina. Encuentran que las condiciones locales del mercado de trabajo desempeñan un papel importante en la explicación de la magnitud de las tasas de salida del desempleo (Arranz, Cid y Muro, 2000), que la flexibilización laboral de los noventa tuvo un impacto importante sobre los flujos de entrada y salida del empleo, incrementando las tasas de salida del empleo (Hopenhayn, 2001), y que, si bien el tiempo corrido que un individuo pasa desempleado es corto, la proporción de tiempo en dos años que éste pasa desempleado es alta debido al fenómeno de reincidencia en el desempleo (Galiani y Hopehayn, 2001). En el presente trabajo se avanza hacia el análisis de la relación entre la tasa de salida del desempleo y el ciclo económico, cuestión no evaluada hasta el momento para la Argentina.

Se estiman modelos para la tasa de salida del desempleo incorporando características socioeconómicas de los individuos y distintas alternativas para captar la incidencia del ciclo económico. También se incluye una medida no paramétrica de la dependencia de la duración. Se encuentra que cuando la economía se expande, la probabilidad de experimentar un tránsito hacia el empleo se incrementa y, viceversa. La probabilidad de salir del desempleo para un individuo promedio que se encuentra entre el tercer y el sexto mes de desempleo es $16,2 \%$ más elevada cuando el producto crece $4 \%$ que cuando cae a igual tasa. También se encuentran diferencias sectoriales significativas.

El trabajo está organizado en seis secciones. En la segunda se presenta sucintamente la evolución de las principales variables del mercado laboral argentino. En la tercera sección se describe el modelo econométrico utilizado y, en la siguiente, se detalla de qué manera se construyó la base de datos utilizada para las estimaciones y se describe brevemente la evolución muestral de la duración del desempleo. La quinta sección contiene el resultado de las regresiones. En la última sección se presentan las consideracio nes finales. 


\section{Los hechos estilizados}

El proceso de reformas estructurales ocurrido durante los 90 en varios países latinoamericanos, y con particular fuerza en la Argentina, ha estado acompañado por un fuerte crecimiento económico y elevados niveles de desocupación. La tasa de desempleo creció de manera importante. En el Gran Buenos Aires $^{2}$ pasó de un valor de $5 \%$ promedio durante la década del 80 a un promedio que rondó el $12 \%$ en los noventa, con picos que alcanzaron el $20 \%$ en mayo de 1995 y el 22\% en mayo de 2002.

\section{Gráfico $\mathbf{N}^{\circ} 1$}

Tasa de desempleo en el GBA ${ }^{(*)}$ y nivel de actividad. 1980-2003

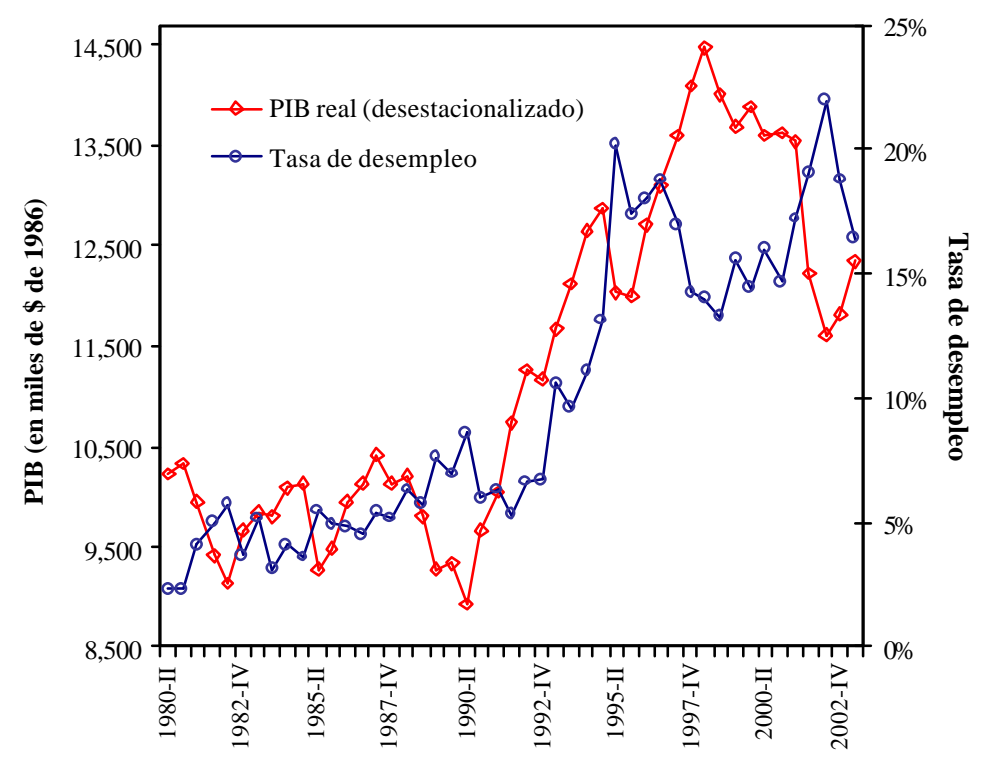

(*) Gran Buenos Aires.

Fuente: elaboración propia en base a INDEC.

Durante los primeros años posteriores a las reformas llevadas a cabo en h Argentina, el crecimiento del producto estuvo acompañado por un aumento de la tasa de desempleo, relación que se revirtió hacia mediados de la década del 90, momento en el que volvió a observarse la relación negativa característica entre nivel de actividad y tasa de desempleo, tal como la prevaleciente, por ejemplo, durante los 80 .

Lo ocurrido durante la primera mitad de la década del 90 es usual en todos los países que llevaron a cabo reformas económicas comparables a la de Argentina (Llach y Llach, 1998). 
Luego de las reformas sobreviene una fase de débil creación de empleo y aumento del desempleo, seguida por una segunda etapa, con la economía ya reestructurada, de fuerte aumento de la ocupación. Obviamente, una condición necesaria para que la segunda etapa se verifique es que la economía mantenga un sendero de crecimiento, lo cual no ocurrió en Argentina, al menos de manera sostenida.

Como es sabido, la tasa de desocupación está determinada por el flujo hacia el desempleo y por la duración promedio de los períodos de desempleo. La misma tasa puede estar asociada con altos flujos y períodos de desocupación relativamente cortos, o con tasas de flujo bajas y largos períodos de desempleo.

\subsection{Flujos hacia el desempleo}

El aumento del desempleo de la primera mitad de la década del 90 se relaciona con la destrucción de empleos (aumento de los despidos) asociada, en parte, con la obsolescencia del capital humano producto del cambio en la demanda laboral por sectores y por calificación que trajo aparejado el proceso de reestructuración económica, en un marco institucional del mercado laboral poco flexible (Pessino, 1996). En menor medida, también contribuyeron en este proceso el aumento de la oferta de trabajo producto del incremento de la participación laboral de la mujer ${ }^{3}$ y la crisis del superpoblado sector informal (Llach y Llach, 1998).

Desde el inicio de la década del 90 y hasta la crisis del Tequila en 1995, tanto el empleo como el desempleo crecieron. Tras la puesta en marcha del plan de Convertibilidad y la progresiva implementación de reformas estructurales (desregulación, apertura comercial y financiera, privatizaciones), la economía argentina se expandió a una tasa promedio de 6,6\% y generó entre mayo de 1990 y mayo de 1994, en términos netos, un total de 279 mil puestos de trabajo. Sin embargo, el incremento del desempleo también fue importante. El número de desocupados se incrementó en 159 mil personas en el mismo lapso.

\footnotetext{
${ }^{2}$ El mercado laboral del Gran Buenos Aires cubre aproximadamente la mitad de la fuerza laboral del país.

${ }^{3}$ Pessino (1996) señala que si bien hubo un aumento significativo en la participación laboral de la mujer, éste se mantuvo dentro de las tendencias mundiales y explica sólo una parte de la mayor desocupación.
} 


\section{Gráfico $\mathbf{N}^{\circ} 2$}

Empleo y desempleo en el $G B A^{(*)}$. 1989-2002

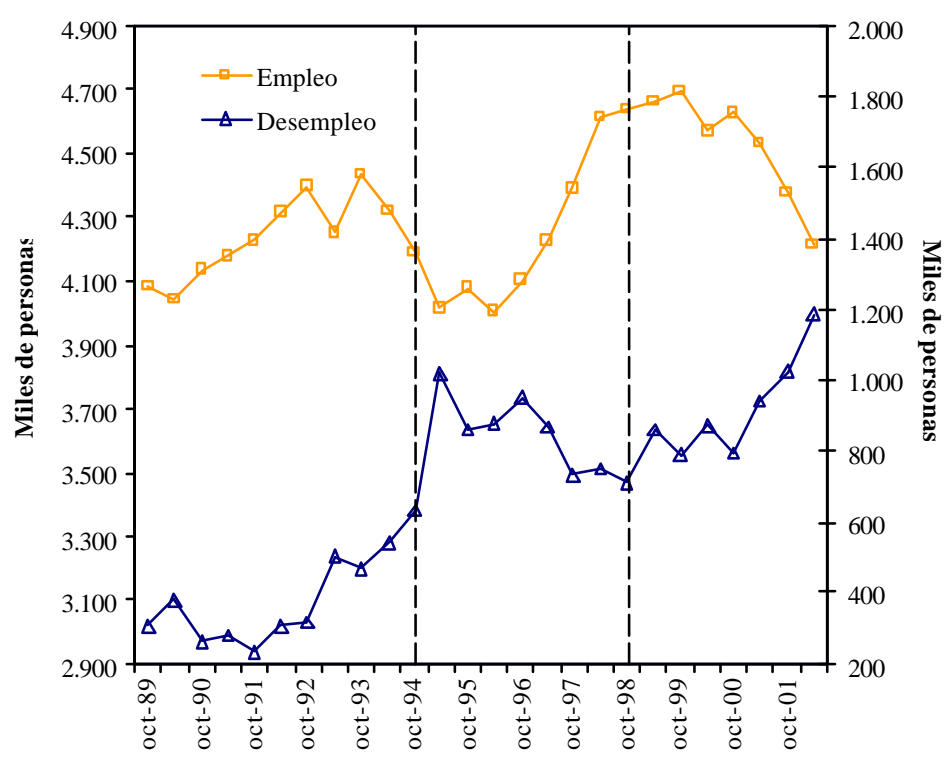

(*) Gran Buenos Aires.

Fuente: elaboración propia en base a INDEC.

Con la crisis mejicana se profundizó el aumento del desempleo y la caída del empleo se hizo pronunciada. Tras la fuerte contracción del nivel de actividad económica, entre 1994 y 1995 el número de desempleados se elevó en 227 mil personas y el número de empleados cayó en 115 mil individuos. La rápida reversión del proceso recesivo dio paso a una nueva fase de crecimiento del empleo, pero esta vez acompañada de una reducción del número de desempleados. Entre mayo de 1996 y mayo de 1998 el empleo se incrementó en 605 mil individuos y, paralelamente, el desempleo se redujo en 129 mil personas. La caída del desempleo que acompañó al crecimiento del producto sugiere que hacia 1996 la economía ya se había reestructurado y que, por lo tanto, la fase de "crecimiento sin empleo" había concluido; si la si el crecimiento económico se mantenía, seguramente el desempleo se hubiera reducido progresivamente.

Sin embargo, la desaceleración de ingreso de capitales tras sucesivos shocks externos (crisis asiática con devaluaciones competitivas, caída de términos de intercambio, fortalecimiento del dólar, devaluación de Brasil) resintió el nivel de actividad económica. El producto se contrajo progresivamente desde el tercer trimestre de 1998 y su retracción se profundizó durante 2001, año en el que colapsó el sistema financiero, Argentina declaró el 
default de su deuda pública y el esquema de Convertibilidad quedó virtualmente desarticulado. En ese marco el desempleo creció a la par de la caída del empleo.

El comportamiento de la tasa de desempleo durante la década del 90 y los primeros años de la siguiente estuvo acompañado por un importante incremento de la rotación laboral.

\section{Gráfico $\mathbf{N}^{\circ} 3$}

Tasa de creación y de destrucción de empleo en el $G B A^{(*)}$. 1989-2002

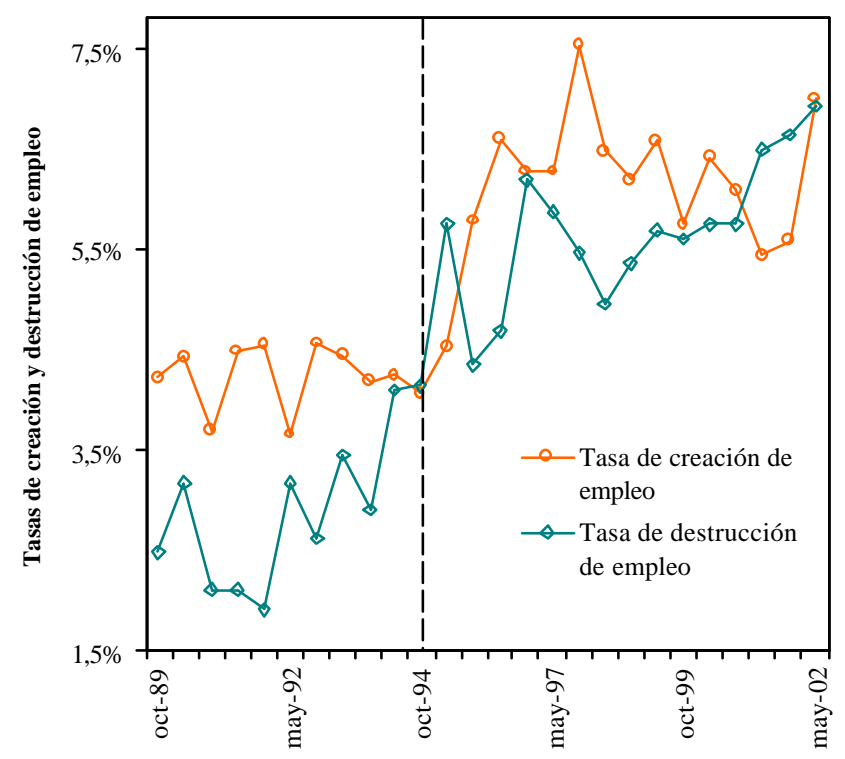

Nota: (a) Tasa de creación de empleo: \% de individuos que llevan 1 mes o menos de empleo/ total de empleados; (b) Tasa de destrucción de empleo: \% de individuos que llevan 1 mes o menos de desempleo / total de empleados.

(*) Gran Buenos Aires.

Fuente: elaboración propia en base a EPH.

Tanto la creación como la destrucción de empleo crecieron, sobre todo a partir de mediados de los 90. Entre los años 1989 y 1994 las tasas de creación y de destrucción de empleo se mantuvieron en valores promedio de $4,2 \%$ y $2,9 \%$, respectivamente. Durante los años siguientes, estas tasas se incrementaron progresivamente hasta alcanzar en ambos casos valores cercanos a 7\%. El punto de quiebre (1995) es un año recesivo, pero también el inicio de un nuevo régimen laboral (contratos temporales). Hopenhayn (2001) encuentra 
que esa reforma tuvo un fuerte impacto sobre la rotación laboral (aumentando tanto la creación como la destrucción de empleos). ${ }^{4}$

\subsection{Duración de los episodios de desempleo}

El desempleo de larga duración, medido como la proporción del total de individuos desempleados que llevan un año o más de desempleo, tuvo un comportamiento similar al de la tasa de desempleo, aumentando fuertemente luego del proceso de reformas económicas.

\section{Gráfico $\mathbf{N}^{\circ} 4$}

Desempleo de larga duración en el $G B A^{(*)}$ y nivel de actividad. 1989-2002

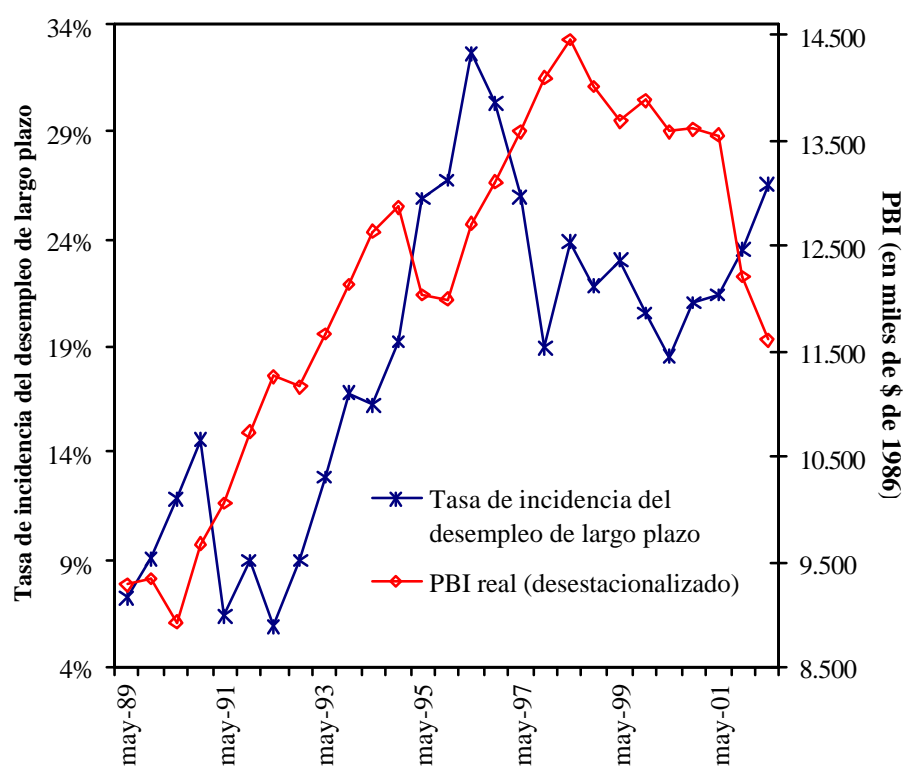

Nota: Tasa de incidencia del desempleo de largo plazo: \% de desempleados con duración de un año o más / total de desempleados.

(*) Gran Buenos Aires.

Fuente: elaboración propia en base a INDEC.

\footnotetext{
4 Elevados flujos pueden tener efectos positivos sobre la eficiencia económica al contribuir a una mejor asignación de recursos, pero también pueden tener costos sociales altos si la rotación es "excesiva", ocasionando pérdida de habilidades específicas para un trabajo determinado y ostos de transacción para trabajadores y empleadores. Cuando el aumento de la rotación laboral se debe al uso de contratos temporarios, si los trabajadores en puestos permanentes permanecen relativamente inmóviles, algunos trabajadores (como los jóvenes y las mujeres) pueden soportar una excesiva proporción de la carga del desempleo.
} 
La tasa de incidencia del desempleo de larga duración creció desde niveles cercanos a 3\% a inicios de la década del 90 hasta tasas superiores al 30\% hacia el año 1996, valor relativamente elevado, aunque considerablemente menor que en el caso europeo (Galiani y Hopenhayn, 2001). El fuerte crecimiento del producto que siguió al proceso de reformas estructurales estuvo acompañado por un importante aumento de la tasa de incidencia del desempleo de largo plazo, lo cual sugiere que la duración del desempleo explica al menos una parte del aumento de la tasa de desempleo.

A partir de mediados de los años noventa se verificó una relación negativa entre nivel de actividad y desempleo de largo plazo y, si bien este último se redujo con el crecimiento del producto que siguió a la crisis del Tequila, se mantuvo en niveles elevados respecto a los primeros años de la década pasada, y volvió a incrementarse con el proceso recesivo iniciado en 1998.

\section{$\underline{\text { Evidencia empírica de la duración del desempleo en la Argentina }}$}

Los estudios sobre los determinantes de la duración del desempleo son abundantes en la literatura económica desde la década del $80 .^{5}$ Los análisis para Argentina son algo más recientes, en parte porque las bases necesarias para realizar las estimaciones están disponibles desde mediados del 90.

Arranz, Cid y Muro (2000) estiman un modelo semiparamétrico discreto para la tasa mensual de salida del desempleo que incluye como variables explicativas una especificación no paramétrica de la dependencia de la duración y un conjunto de características personales y económicas. La muestra está construida a partir de un panel conformado con la Encuesta Permanente de Hogares (EPH) para los años 1997 y 1998.

La estimaciones sugieren que las condiciones locales del mercado de trabajo desempeñan un papel importante en la explicación de la magnitud de las tasas de salida del desempleo en la Argentina, ${ }^{6}$ con la Ciudad de Buenos Aires como el lugar que presenta las mejores

\footnotetext{
${ }^{5}$ Para un compendio de esta literatura ver Atkinson y Micklewright (1991).

${ }^{6}$ Varios autores han enfatizado la existencia de diferencias regionales en la problemática del desemp leo en Argentina. Díaz Cafferata y otros (1997) señalan que se observan diferencias regionales en el ritmo de crecimiento de la oferta de trabajo. Lamarche y otros (1998) encuentran la existencia de factores locales
} 
condiciones para salir del desempleo. Encuentran una relación directa entre la tasa de salida del desempleo y el nivel de capital humano del individuo, y una relación inversa entre las condiciones familiares adversas (en términos de estado de actividad del jefe del hogar y el número de integrantes desempleados) y la tasa de salida del desempleo. No encuentran efectos fijos en el tiempo.

Hopenhayn (2001) analiza la incidencia de la flexibilización de los noventa (reformas del $91^{7}$ y del 95) sobre la duración del empleo. Durante los noventa ha habido cambios en la legislación laboral a partir de la introducción de flexibilidad en el margen creando contratos de período fijo y temporales (“de prueba") que eliminan o reducen los costos de despido y los impuestos al trabajo. Los resultados que obtiene el autor sugieren que tales cambios tuvieron un impacto importante sobre los flujos de entrada y salida del empleo, incrementando las tasas de riesgo de salida del empleo ${ }^{8}$ durante el período de prueba (los primeros tres meses de empleo) en un $40 \%$.

Galiani y Hopenhayn (2001) estimaron las tasas de riesgo de salir del empleo y de salir del desempleo en función de características personales del individuo (sexo, edad, nivel educativo) permitiendo dependencia de la duración. Las tasas de riesgo de salida del empleo y del desempleo se reducen con la edad. Un mayor nivel educativo reduce monotónicamente la probabilidad de salir del empleo. En ambos casos se encuentra una fuerte dependencia de la duración ne gativa. Finalmente, la tasa de salida del empleo se incrementa considerablemente con la introducción de contratos laborales más flexibles en 1995.

Luego, estiman el riesgo de desempleo que enfrenta un individuo desempleado en un período de dos años y encue ntran que si bien el tiempo corrido que éste pasa desempleado es corto, la proporción de tiempo en dos años que pasa desempleado es alta debido al fenómeno de reincidencia en el desempleo. En particular, hallan que un individuo promedio que ingresa en el desempleo en 1998 experimenta 3 períodos de desocupación en los

específicos en la determinación del desempleo. Arrufat y otros (1999) también hayan diferencias regionales y persistencia de niveles altos de desocupación.

${ }^{7}$ Esta reforma no tuvo un gran impacto dado que no alcanzó a una gran cantidad de trabajadores. 
siguientes dos años y acumula en ese lapso de tiempo un total de 6,3 meses sin empleo. A su vez, estiman que la reincidencia en el desempleo se incrementó 50\% entre 1989 y 1998, y la duración media del desempleo acumulada en dos años un $43 \%$ en el mismo período.

En el presente trabajo se avanza hacia el análisis de la relación entre las tasa de salida del desempleo y el ciclo económico, cuestión no evaluada hasta el momento para la Argentina. ${ }^{9}$ Para ello se estima un modelo de duración del desempleo que incluye, además de características socioeconómicas, distintas medidas de la evolución del ciclo para los años cubiertos por la muestra.

${ }^{8}$ Se define como la probabilidad de salir del empleo condicional a que el individuo ha transcurrido determinada cantidad de tiempo en el empleo.

${ }^{9}$ Existen estudios para otros países sobre este tema (ver, por ejemplo, Bover, Arellano, y Bentolila (1996) para una discusión del caso español). 


\section{El modelo econométrico}

Se construyó un modelo discreto ${ }^{10}$ para la probabilidad de que un individuo salga del desempleo en el transcurso del mes siguiente al mes de desempleo considerado, teniendo en cuenta que no ha encontrado trabajo en determinada cantidad de meses. La variable de interés es el tiempo que tarda un individuo en pasar del desempleo al empleo, ${ }^{11}$ de modo que el suceso que marca la transición de un estado a otro es "encontrar trabajo".

La función de riesgo que se utiliza en este trabajo es la log-log complementaria, una alternativa usual dentro de los modelos discretos de duración. ${ }^{12}$ Sea $z(t)=c(t)+X \beta$ para un individuo representativo en el período $t$, donde $c(t)$ es la función de riesgo base y $X \beta$ incluye el intercepto, la función de riesgo en tiempo discreto log-log complementaria será $p(t)$, donde:

$$
\begin{gathered}
\log [-\log (1-p(t))]=z(t) \quad \text { luego, } \\
p(t)=1-\exp [-\exp (z(t))]
\end{gathered}
$$

Para la función de riesgo base, $c(t)$, se eligió una especificación no paramétrica, a partir de la construcción de variables dicotómicas para intervalos de duración del desempleo. La especificación del coeficiente lineal, $X \beta$, incluye características socioeconómicas de los individuos: sexo, edad y nivel educativo; y diversas alternativas para medir las condiciones macroeconómicas del período abarcado por muestra: tasa de crecimiento del producto, tasas de desempleo sectorial y ciclo económico. Cada una de estas alternativas para captar el estado del ciclo económico da origen a un modelo distinto (ver Tabla $N^{\circ} 2$ en la sección 5).

\footnotetext{
${ }^{10}$ Se elig ió un modelo discreto porque las duraciones observadas son mensuales.

${ }^{11}$ No se consideran las transiciones del desempleo a la inactividad. Existen al menos dos razones para ello: (a) no se dispone de información estadística suficiente para calcular la duración del desempleo cuando el individuo transita del desempleo hacia la inactividad (ver más adelante), y (b) es una práctica usual en la literatura empírica sobre el tema (ver por ejemplo Galiani y Hopenhayn (2001)). Sin embargo, debe tenerse en cuenta que, dado que el período bajo análisis se caracteriza por elevados flujos laborales, la omisión de las transiciones del desempleo a la inactividad puede ser potencialmente importante.
} 


\section{La construcción de los datos}

La base de datos se construyó a partir de paneles rotatorios obtenidos de la EPH que elabora el INDEC con periodicidad semestral. ${ }^{13}$ La información abarca el período mayo de 1995 - mayo de $2003 .{ }^{14}$ En cada onda de la encuesta se renueva el $25 \%$ de la muestra, de modo que algunos individuos son observados hasta cuatro veces en un período calendario de dos años.

Esta encuesta provee información acerca del estado de actividad de los individuos entrevistados (empleado, desempleado, inactivo), del tiempo que han permanecido en el empleo o en el desempleo (no así en la inactividad), y de diversas características socioeconómicas de cada uno de ellos.

Con la información retrospectiva disponible y con el seguimiento temporal que realiza la encuesta, es posible conocer el tiempo exacto que han estado desempleados varios de los individuos entrevistados. Tomemos el caso de una persona encuestada dos veces en forma consecutiva, en el momento $t$ y en el $t+6$, respectivamente. Supongamos que en la primera entrevista esta persona estaba desempleada, y que al momento de la segunda se encuentra empleada. A partir de la primera encuesta puede conocerse la cantidad de meses que el individuo estuvo desempleado hasta $t, d_{t}$. Luego, la duración del desempleo para este individuo hasta $t$ será $D_{t}=d_{t}$. Utilizando la segunda encuesta pueden determinarse los meses que el individuo ha estado empleado hasta el período $t+6, e_{t+6}$. Nótese que $e_{t+6}$ debe ser necesariamente menor o igual que 6 , dado que ese es el número de meses que separan a la segunda encuesta de la primera, en la cual el individuo declaró estar desempleado hacía $d_{t}$ meses. De este modo, dado que éste ha experimentado una transición del desempleo al empleo, la duración completa del desempleo para este individuo observada en el período $t+6$ será $D_{t+6}=d_{t}+6-e_{t+6}$.

\footnotetext{
${ }^{12}$ Ver al respecto Jenkins (2004).

${ }^{13}$ El primer relevamiento del año se realiza en el mes de mayo y el segundo en el mes de octubre.

${ }^{14}$ Las encuestas anteriores a 1995 no incluyen en la base usuario la variable que permite identificar a los individuos en las diferentes ondas, de modo que para esas encuestas no es posible construir de manera directa sus historias de desempleo (ver más adelante).
} 
Obviamente, en el caso de aquellos individuos desempleados para los cuales se dispone de información para un único momento en el tiempo o aquellos que durante el período en el que han sido entrevistados continúan desempleados, no será posible conocer la duración completa del período de desempleo. Sólo es posible establecer que como mínimo han estado desempleados una determinada cantidad de tiempo; a los efectos de este trabajo se trata de observaciones censuradas por la derecha.

Para estimar un modelo discreto como el descripto en la sección anterior es necesario organizar la base de datos de forma tal que se tengan tantas observaciones por individuo como períodos de tiempo éste haya estado "en riesgo", es decir, una por período de desempleo. También es necesario identificar aquellos períodos de desempleo incluidos en la base que finalizan en una transición hacia el empleo.

La muestra está compuesta por individuos inicialmente desempleados, con edades comprendidas entre los 21 y los 65 años de edad, que no hayan estado desempleados más de 48 meses. También fueron eliminadas aquellas personas observadas en una sola encuesta. $^{15}$

\section{Descripción de los datos de duración}

En la tabla siguiente se resumen las características principales de los datos de duración utilizados y se presentan algunas estadísticas descriptivas de la duración muestral del desempleo, distinguiendo entre períodos de expansión y de recesión económica.

\footnotetext{
${ }^{15}$ Este tipo de restricciones de la muestra son usuales en los trabajos que estiman modelos similares (ver por ejemplo Bover, Arellano y Bentolila (1996), Arranz, Cid y Muro (2000), Hopenhayn (2001)).
} 
Tabla $\mathrm{N}^{\circ} \mathbf{1}$

Descripción de los datos de duración del desempleo

\begin{tabular}{|c|c|c|c|}
\hline \begin{tabular}{|c|}
$\begin{array}{c}\text { Meses de } \\
\text { desempleo }\end{array}$ \\
$(1)$
\end{tabular} & \begin{tabular}{|c|} 
Número de \\
individuos \\
en riesgo \\
$(2)$
\end{tabular} & $\begin{array}{c}\text { Número de tránsitos } \\
\text { del desempleo al } \\
\text { empl eo } \\
\text { (3) }\end{array}$ & $\begin{array}{l}\text { Número de } \\
\text { individuos } \\
\text { censurados } \\
(4)\end{array}$ \\
\hline 1 & 4.094 & 63 & 681 \\
\hline 2 & 3.350 & 46 & 205 \\
\hline 3 & 3.099 & 61 & 168 \\
\hline 4 & 2.870 & 86 & 90 \\
\hline 5 & 2.694 & 131 & 81 \\
\hline 6 & 2.482 & 196 & 104 \\
\hline 7 & 2.182 & 107 & 216 \\
\hline 8 & 1.859 & 91 & 129 \\
\hline 9 & 1.639 & 69 & 96 \\
\hline 10 & 1.474 & 55 & 71 \\
\hline 11 & 1.348 & 71 & 52 \\
\hline 12 & 1.225 & 75 & 178 \\
\hline 13 & 972 & 33 & 83 \\
\hline 14 & 856 & 25 & 49 \\
\hline 15 & 782 & 37 & 32 \\
\hline 16 & 713 & 32 & 35 \\
\hline 17 & 646 & 46 & 22 \\
\hline 18 & 578 & 27 & 133 \\
\hline 19 & 418 & 9 & 30 \\
\hline 20 & 379 & 6 & 21 \\
\hline 21 & 352 & 13 & 20 \\
\hline 22 & 319 & 12 & 10 \\
\hline 23 & 297 & 17 & 5 \\
\hline 24 & 275 & 14 & 93 \\
\hline 25 & 168 & 2 & 3 \\
\hline 26 & 163 & 5 & 7 \\
\hline 27 & 151 & 8 & 4 \\
\hline 28 & 139 & 2 & 1 \\
\hline 29 & 136 & 13 & 0 \\
\hline 30 & 123 & 1 & 35 \\
\hline 32 & 87 & 0 & 2 \\
\hline 34 & 85 & 3 & 0 \\
\hline 35 & 82 & 4 & 0 \\
\hline 36 & 78 & 4 & 36 \\
\hline 38 & 38 & 1 & 0 \\
\hline 39 & 37 & 5 & 0 \\
\hline 40 & 32 & 2 & 0 \\
\hline 41 & 30 & 1 & 0 \\
\hline 42 & 29 & 1 & 13 \\
\hline 43 & 15 & 0 & 1 \\
\hline 47 & 14 & 1 & 0 \\
\hline 48 & 13 & 0 & 13 \\
\hline
\end{tabular}

Total de individuos

Total de tránsitos del desempleo al empleo Total de individuos censurados
4.094

1.375

2.719

\begin{tabular}{|c|c|c|}
\hline $\begin{array}{c}\text { Función de } \\
\text { supervivencia } \\
\text { (agregada) } \\
(5)\end{array}$ & \begin{tabular}{|c|} 
Función de \\
supervivencia \\
(en expansión) \\
$(6)$
\end{tabular} & $\begin{array}{c}\text { Función de } \\
\text { supervivencia } \\
\text { (en recesión) } \\
\text { (7) }\end{array}$ \\
\hline 0,9846 & 0,9765 & 0,9879 \\
\hline 0,9711 & 0,9565 & 0,9771 \\
\hline 0,9520 & 0,9355 & 0,9588 \\
\hline 0,9235 & 0,9033 & 0,9319 \\
\hline 0,8785 & 0,8613 & 0,8857 \\
\hline 0,8092 & 0,7870 & 0,8185 \\
\hline 0,7695 & 0,7438 & 0,7803 \\
\hline 0,7318 & 0,7029 & 0,7439 \\
\hline 0,7010 & 0,6704 & 0,7139 \\
\hline 0,6749 & 0,6411 & 0,6891 \\
\hline 0,6393 & 0,5936 & 0,6592 \\
\hline 0,6002 & 0,5566 & 0,6191 \\
\hline 0,5798 & 0,5366 & 0,5987 \\
\hline 0,5629 & 0,5208 & 0,5813 \\
\hline 0,5362 & 0,4942 & 0,5548 \\
\hline 0,5122 & 0,4795 & 0,5258 \\
\hline 0,4757 & 0,4375 & 0,4927 \\
\hline 0,4535 & 0,3980 & 0,4801 \\
\hline 0,4437 & 0,3892 & 0,4699 \\
\hline 0,4367 & 0,3798 & 0,4643 \\
\hline 0,4206 & 0,3534 & 0,4545 \\
\hline 0,4047 & 0,3322 & 0,4421 \\
\hline 0,3816 & 0,3103 & 0,4185 \\
\hline 0,3621 & 0,2881 & 0,4009 \\
\hline 0,3578 & 0,2881 & 0,3938 \\
\hline 0,3469 & 0,2718 & 0,3866 \\
\hline 0,3285 & 0,2505 & 0,3711 \\
\hline 0,3238 & 0,2505 & 0,3630 \\
\hline 0,2928 & 0,2239 & 0,3304 \\
\hline 0,2904 & 0,2187 & 0,3304 \\
\hline 0,2904 & 0,2187 & 0,3304 \\
\hline 0,2802 & 0,2041 & 0,3244 \\
\hline 0,2665 & 0,1968 & 0,3063 \\
\hline 0,2528 & 0,1892 & 0,2887 \\
\hline 0,2462 & 0,1892 & 0,2766 \\
\hline 0,2129 & 0,1487 & 0,2526 \\
\hline 0,1996 & 0,1352 & 0,2406 \\
\hline 0,1930 & 0,1352 & 0,2285 \\
\hline 0,1863 & 0,1352 & 0,2158 \\
\hline 0,1863 & 0,1352 & 0,2158 \\
\hline 0,1730 & 0,1081 & 0,2158 \\
\hline 0,1730 & 0,1081 & 0,2158 \\
\hline
\end{tabular}

Nota: sea $t$ el mes de desempleo, la función de Supervivencia será: $\prod_{i=1}^{t}\left(1-\frac{\text { Columna }(3)_{i}}{\text { Columna }(2)_{i}}\right)$. 
En la primera columna se presenta el sendero de desempleo medido en meses. El número 1 indica el primer mes de desempleo del individuo, el número 2 el segundo, y así sucesivamente hasta llegar al número 48, máxima cantidad de meses continuos que un individuo transita por el desempleo en esta muestra.

La cantidad total de personas incluidos en la muestra es 4.094, número de individuos que inicialmente se encuentra "en riesgo" de transitar del desempleo al empleo (segunda fila de la segunda columna de la tabla). Al iniciar el segundo mes de desempleo, se observa que 63 individuos encontraron un empleo (segunda fila de la tercera columna) y 681 individuos salieron de la muestra pero no se tiene información acerca de si experimentaron una transición hacia el empleo (segunda fila de la cuarta columna). En este último caso se trata de observaciones censuradas por la derecha. Dados estos movimientos, durante el segundo mes de desempleo quedan 3.350 individuos "en riesgo". ${ }^{16}$ Siguiendo un razonamiento análogo pueden leerse las filas siguientes.

Del total de individuos incluidos en la muestra, 1.375 experimentaron una transición observada del desempleo al empleo, esto es el 33,6\%. Los 2.719 individuos restantes salieron de la muestra sin que se tenga información acerca de si experimentaron o no una transición.

Con la información de las columnas (2), (3) y (4) de la tabla anterior se calculó un indicador que usualmente se utiliza para realizar una descripción preliminar de la dinámica de la duración del desempleo: la función de supervivencia (columna (5)). Sea $t$ el mes de desempleo del individuo, $T_{t}$ el número de tránsitos del desempleo al empleo en $t$ y $R_{t}$ el número de individuos en riesgo en $t$, la función de supervivencia del período $t, S(t)$, se define como:

$$
S(t)=\prod_{i=1}^{t}\left(1-\frac{T_{i}}{R_{i}}\right)
$$

Esta función mide la probabilidad de que el individuo permanezca en el desempleo en cada uno de los meses de tránsito en ese estado. Las columnas (6) y (7) contienen las funciones de supervivencia correspondientes a los meses de expansión y recesión económica, 
respectivamente. Las tres funciones de supervivencia se representan gráficamente a continuación.

\section{Gráfico $\mathbf{N}^{\circ} 5$}

Funciones de supervivencia

Panel (a)
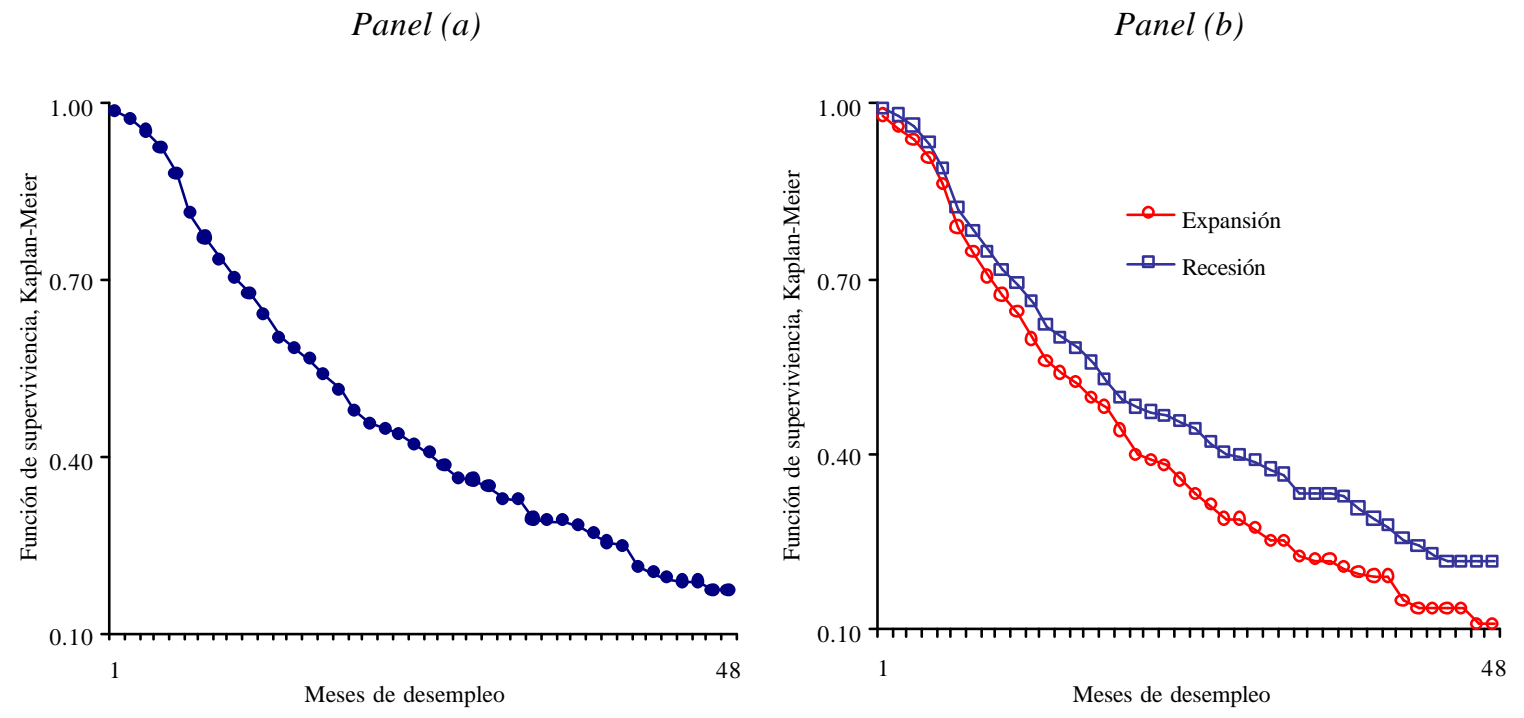

Como es usual, la probabilidad de permanecer en el desempleo se reduce con la duración del mismo (Gráfico $\mathrm{N}^{\circ}$ 5, Panel (a)). En el primer mes de desempleo la función de supervivencia es cercana a 1 . Luego de seis meses de desempleo su valor se reduce a algo más de 0,8 y en el transcurso de un año a 0,6. La duración mediana del desempleo (probabilidad de supervivencia igual a 0,5) se encuentra entre 16 y 17 meses. Después de 48 meses de desempleo (duración máxima de la muestra) todavía existe una probabilidad de 0,17 de no experimentar un tránsito hacia el empleo.

La función de supervivencia calculada para períodos de expansión económica se encuentra por debajo de la correspondiente a períodos recesivos para todos los meses de desempleo, indicando que la probabilidad de continuar desempleado el mes siguiente es menor en el primer caso (Gráfico $\mathrm{N}^{\circ}$ 5, Panel (b)). A su vez, la diferencia entre ambas funciones tiende a elevarse conforme aumenta el número de meses de desempleo. Estos resultados sugieren que el ciclo económico importa para la duración del desempleo.

\footnotetext{
${ }^{16}$ Esto es: $4.094-63-681$.
} 


\section{Los resultados econométricos}

En la tabla siguiente se presentan los resultados de las estimaciones de los modelos de duración del desempleo, incorporando diferentes especificaciones para captar el efecto del ciclo económico.

Tabla $\mathbf{N}^{\circ} 2$

Resultados de las diferentes especificaciones del modelo de duración

\begin{tabular}{|c|c|c|c|c|c|}
\hline Variables & Modelo 1 & Modelo 2 & Modelo 3 & Modelo 4 & Modelo 5 \\
\hline \multicolumn{6}{|c|}{ Características socioeconómicas } \\
\hline 1 si varón y 0 si mujer & $\begin{array}{c}0,2960 * * * \\
(0,0000)\end{array}$ & $\begin{array}{c}0,3181 * * * \\
(0,0000)\end{array}$ & $\begin{array}{c}0,3234 * * * \\
(0,0000)\end{array}$ & $\begin{array}{c}0,3237 * * * \\
(0,0000)\end{array}$ & $\begin{array}{c}0,2817 * * * \\
(0,0000)\end{array}$ \\
\hline Edad & $\begin{array}{r}0,0398 * * \\
(0,0190)\end{array}$ & $\begin{array}{c}0,0446 * * * \\
(0,0090)\end{array}$ & $\begin{array}{c}0,0450 * * * \\
(0,0080)\end{array}$ & $\begin{array}{c}0,0448 * * * \\
(0,0080)\end{array}$ & $\begin{array}{c}0,0421 * * \\
(0,0150)\end{array}$ \\
\hline Edad al cuadrado & $\begin{array}{c}-0,0007 * * * \\
(0,0010)\end{array}$ & $\begin{array}{c}-0,0008 * * * \\
(0,0000)\end{array}$ & $\begin{array}{c}-0,0008 * * * \\
(0,0000)\end{array}$ & $\begin{array}{c}-0,0008 * * * \\
(0,0000)\end{array}$ & $\begin{array}{c}-0,0007 * * * \\
(0,0010)\end{array}$ \\
\hline 1 si educación primaria completa ${ }^{(1)}$ & $\begin{array}{l}-0,0791 \\
(0,3490)\end{array}$ & $\begin{array}{c}-0,0903 \\
(0,2850)\end{array}$ & $\begin{array}{l}-0,0818 \\
(0,3330)\end{array}$ & $\begin{array}{c}-0,0791 \\
(0,3490)\end{array}$ & $\begin{array}{l}-0,0347 \\
(0,6870)\end{array}$ \\
\hline 1 si educación secundaria incompleta & $\begin{array}{c}-0,2282 * * \\
(0,0170)\end{array}$ & $\begin{array}{c}-0,2068 * * \\
(0,0310)\end{array}$ & $\begin{array}{c}-0,2042 * * \\
(0,0340)\end{array}$ & $\begin{array}{c}-0,2031 * * \\
(0,0350)\end{array}$ & $\begin{array}{l}-0,1329 \\
(0,1830)\end{array}$ \\
\hline 1 si educación secundaria completa & $\begin{array}{c}-0,4127 * * * \\
(0,0000)\end{array}$ & $\begin{array}{c}-0,3995 * * * \\
(0,0000)\end{array}$ & $\begin{array}{c}-0,3935 * * * \\
(0,0000)\end{array}$ & $\begin{array}{c}-0,3925 * * * \\
(0,0000)\end{array}$ & $\begin{array}{c}-0,3062 * * * \\
(0,0040)\end{array}$ \\
\hline 1 si educación universitaria incompleta & $\begin{array}{c}-0,3005 * * \\
(0,0140)\end{array}$ & $\begin{array}{c}-0,2866^{* *} \\
(0,0190)\end{array}$ & $\begin{array}{c}-0,2810 * * \\
(0,0210)\end{array}$ & $\begin{array}{c}-0,2819 * * \\
(0,0210)\end{array}$ & $\begin{array}{l}-0,1805 \\
(0,1580)\end{array}$ \\
\hline 1 si educación universitaria completa & $\begin{array}{c}-0,3032 * * \\
(0,0390)\end{array}$ & $\begin{array}{c}-0,3418 * * \\
(0,0200)\end{array}$ & $\begin{array}{c}-0,3457 * * \\
(0,0180)\end{array}$ & $\begin{array}{c}-0,3453 * * \\
(0,0180)\end{array}$ & $\begin{array}{l}-0,2858 * \\
(0,0630)\end{array}$ \\
\hline \multicolumn{6}{|c|}{ Ciclo económico } \\
\hline \multirow[t]{2}{*}{1 si año $1995^{(2)}$} & $-1,7254 * * *$ & - & - & - & - \\
\hline & $(0,0000)$ & - & - & - & - \\
\hline \multirow[t]{2}{*}{1 si año 1996} & $-0,7426 * * *$ & - & - & - & - \\
\hline & $(0,0000)$ & - & - & - & - \\
\hline \multirow[t]{2}{*}{1 si año 1997} & $-0,4355 * * *$ & - & - & - & - \\
\hline & $(0,0000)$ & - & - & - & - \\
\hline \multirow[t]{2}{*}{1 si año 1998} & $-0,3602 * * *$ & - & - & - & - \\
\hline & $(0,0030)$ & - & - & - & - \\
\hline \multirow[t]{2}{*}{1 si año 1999} & $-0,5733 * * *$ & - & - & - & - \\
\hline & $(0,0000)$ & - & - & - & - \\
\hline \multirow[t]{2}{*}{1 si año 2000} & $-0,5054 * * *$ & - & - & - & - \\
\hline & $(0,0000)$ & - & - & - & - \\
\hline \multirow[t]{2}{*}{1 si año 2001} & $-0,8955 * * *$ & - & - & - & - \\
\hline & $(0,0000)$ & - & - & - & - \\
\hline \multirow[t]{2}{*}{1 si año 2002} & $-0,7808 * * *$ & - & - & - & - \\
\hline & $(0,0000)$ & - & - & - & - \\
\hline \multirow[t]{2}{*}{1 si período de vigencia plan $\mathrm{Jy}^{(3)}$} & - & $0,2089 * *$ & $0,5056 * * *$ & $0,4843 * * *$ & $0,3201 * * *$ \\
\hline & - & $(0,0150)$ & $(0,0000)$ & $(0,0000)$ & $(0,0000)$ \\
\hline \multirow[t]{2}{*}{ Variación del PBI trimestral } & - & $0,0193 * * *$ & - & - & - \\
\hline & - & $(0,0000)$ & - & - & - \\
\hline \multirow[t]{2}{*}{ Ciclo económico ${ }^{(4)}$} & - & - & $0,0191 * * *$ & - & - \\
\hline & - & - & $(0,0000)$ & - & - \\
\hline
\end{tabular}

Continúa.... 
Continuación

\begin{tabular}{|c|c|c|c|c|c|}
\hline Variables & Modelo 1 & Modelo 2 & Modelo 3 & Modelo 4 & Modelo 5 \\
\hline \multicolumn{6}{|c|}{ Ciclo económico (continuación) } \\
\hline \multirow[t]{2}{*}{1 si expansión * Ciclo económico } & - & - & - & $0,0318 * *$ & - \\
\hline & - & - & - & $(0,0110)$ & - \\
\hline \multirow[t]{2}{*}{1 si recesión * Ciclo económico } & - & - & - & $0,0159 * * *$ & - \\
\hline & - & - & - & $(0,0010)$ & - \\
\hline \multirow{2}{*}{$\begin{array}{l}1 \text { si último empleo en sector primario } \\
* \text { tasa de desempleo de ese sector } \\
*(5)\end{array}$} & - & - & - & - & $-0,0393$ \\
\hline & - & - & - & - & $(0,5930)$ \\
\hline \multirow{2}{*}{$\begin{array}{l}1 \text { si último empleo en sector industrial } \\
\text { * tasa de desempleo de ese sector }\end{array}$} & - & - & - & - & $-0,0491 * * *$ \\
\hline & - & - & - & - & $(0,0000)$ \\
\hline \multirow{2}{*}{$\begin{array}{l}1 \text { si último empleo en sector servicios } \\
\text { * tasa de desempleo de ese sector }\end{array}$} & - & - & - & - & $-0,0769 * * *$ \\
\hline & - & - & - & - & $(0,0000)$ \\
\hline \multirow{2}{*}{$\begin{array}{l}1 \text { si último empleo en sector construc- } \\
\text { ción * tasa de desempleo de ese sector }\end{array}$} & - & - & - & - & $-0,0210 * * *$ \\
\hline & - & - & - & - & $(0,0000)$ \\
\hline \multicolumn{6}{|c|}{ Dependencia de la duración } \\
\hline 1 si entre 0 y 3 meses de desempleo & $\begin{array}{c}-3,9233 * * * \\
(0,0000)\end{array}$ & $\begin{array}{l}-4,6873 * * * \\
(0,0000)\end{array}$ & $\begin{array}{c}-4,6502 * * * \\
(0,0000)\end{array}$ & $\begin{array}{c}-4,6802 * * * \\
(0,0000)\end{array}$ & $\begin{array}{c}-3,8440 * * * \\
(0,0000)\end{array}$ \\
\hline 1 si entre 3 y 6 meses de desempleo & $\begin{array}{c}-2,7399 * * * \\
(0,0000)\end{array}$ & $\begin{array}{c}-3,5063 * * * \\
(0,0000)\end{array}$ & $\begin{array}{c}-3,4677 * * * \\
(0,0000)\end{array}$ & $\begin{array}{c}-3,4978 * * * \\
(0,0000)\end{array}$ & $\begin{array}{c}-2,6626 * * * \\
(0,0000)\end{array}$ \\
\hline 1 si entre 6 y 12 meses de desempleo & $\begin{array}{c}-2,8062 * * * \\
(0,0000)\end{array}$ & $\begin{array}{c}-3,5645^{* * *} * \\
(0,0000)\end{array}$ & $\begin{array}{c}-3,5203 * * * \\
(0,0000)\end{array}$ & $\begin{array}{c}-3,5514 * * * \\
(0,0000)\end{array}$ & $\begin{array}{c}-2,7239 * * * \\
(0,0000)\end{array}$ \\
\hline 1 si entre 12 y 24 meses de desempleo & $\begin{array}{c}-2,9614 * * * \\
(0,0000)\end{array}$ & $\begin{array}{l}-3,6909 * * * \\
(0,0000)\end{array}$ & $\begin{array}{c}-3,6486 * * * \\
(0,0000)\end{array}$ & $\begin{array}{l}-3,6804 * * * \\
(0,0000)\end{array}$ & $\begin{array}{c}-2,8204 * * * \\
(0,0000)\end{array}$ \\
\hline 1 si más de 24 meses de desempleo & $\begin{array}{c}-3,1618 * * * \\
(0,0000) \\
\end{array}$ & $\begin{array}{c}-3,8766^{* * *} \\
(0,0000) \\
\end{array}$ & $\begin{array}{c}-3,8382 * * * \\
(0,0000) \\
\end{array}$ & $\begin{array}{c}-3,8735 * * * \\
(0,0000) \\
\end{array}$ & $\begin{array}{c}-3,0423 * * * \\
(0,0000) \\
\end{array}$ \\
\hline \multirow{3}{*}{$\begin{array}{l}\text { Número de observaciones } \\
\text { Wald } \chi^{2}\end{array}$} & 36.026 & 36.575 & 36.575 & 36.575 & 35.480 \\
\hline & 13408,46 & 13754,22 & 13747,05 & 13745,52 & 13317,78 \\
\hline & $(0,0000)$ & $(0,0000)$ & $(0,0000)$ & $(0,0000)$ & $(0,0000)$ \\
\hline
\end{tabular}

Referencias: $* * *$ variable significativa al nivel de $1 \%$; **variable significativa al nivel de $5 \%$; *variable significativa al nivel de 10\%. Entre paréntesis se presentan los valores p del test de significatividad individual de los coeficientes; (1) la categoría base es "educación primaria incompleta"; (2) la categoría base es el año 2003; (3) "JyJ" hace referencia al plan de empleo "Jefes y Jefas de Hogar Desocupados"; (4) diferencia entre el PBI trimestral desestacionalizado y su tendencia calculada con el filtro Hodrick-Prescott; (5) la categoría base es "trabajadores nuevos" (que por lo tanto no pueden asignarse a ningún sector).

Nota: En todos los casos la variables dicotómicas toman los valores 1 y 0.

Las variables socioeconómicas son en general altamente significativas. Los varones tienen una mayor probabilidad de salir del desempleo que las mujeres. Por ejemplo, para dos individuos promedio ${ }^{17}$ que sólo difieren entre sí en el sexo, la probabilidad de salir del desempleo es 33,2\% más alta para el varón que para la mujer. La existencia de diferencias entre ambos sexos puede deberse a dos cuestiones que actúan en el mismo sentido. Por un lado, los varones suelen tener un salario de reserva menor que las mujeres debido a que, en general, son el sostén del hogar, por lo que se ven obligados a aceptar las primeras

${ }^{17}$ Edad promedio muestral (37,8 años), con educación universitaria completa, para el año 1998, que se encuentra entre el tercer y el sexto mes de desempleo. 
propuestas laborales que reciben. Por otro lado, es posible que exista alguna forma de discriminación en contra de las mujeres ${ }^{18}$ que determine que los varones tengan una mayor cantidad de alternativas laborales que estas últimas.

La relación entre la edad y la probabilidad de salir del desempleo tiene una forma cuadrática estadísticamente significativa, tal como puede observarse en el Gráfico $\mathrm{N}^{\circ} 6$ a partir de la representación de la incidencia de esta variable sobre el coeficiente lineal de regresión. ${ }^{19}$ En general, los individuos más jóvenes tienen una probabilidad más elevada de salir del desempleo que aquellos de mayor edad. El valor máximo se da para individuos de 29 años. Un varón promedio ${ }^{20}$ con esa edad tiene una probabilidad 35,4\% mayor de salir del desempleo que uno de 50 años de edad. Este resultado es intuitivo, pues los individuos más jóvenes suelen tener mayor cantidad de oportunidades laborales que los de mayor edad, quienes usualmente tienen dificultades para reinsertarse en el mercado laboral una vez que han caído en el desempleo.

\section{Gráfico $\mathrm{N}^{\circ} 6$}

Relación de la duración del desempleo con el nivel educativo y con la edad
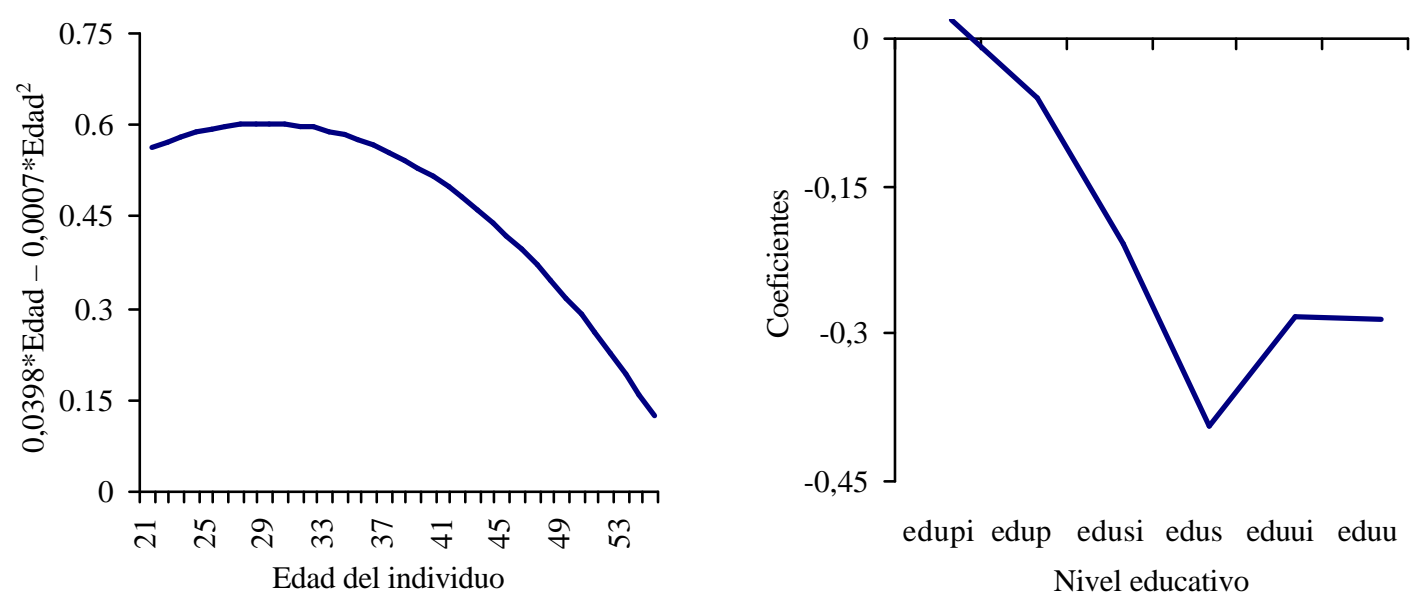

Nota: edupi: educación primaria incompleta; edup: educación primaria completa; edusi: educación secundaria incompleta; edus: educación secundaria completa; eduui: educación universitaria incompleta; eduu: educación universitaria completa.

\footnotetext{
${ }^{18}$ Existe evidencia de hay diferencias salariales a favor del varón que se explican por discriminación de sexos (ver al respecto Gasparini y otros (2002)).

${ }^{19}$ Más específicamente, en el Gráfico $\mathrm{N}^{\circ} 6$ se representa en el eje vertical: $0,0398 * \operatorname{Edad}-0,0007 * \mathrm{Edad}^{2}$.

${ }^{20}$ Idem nota al pie 17 excepto por la edad.
} 
Las variables dicotómicas que captan nivel educativo muestran una relación no monotónica con la probabilidad de salir del desempleo. Esto es lo que se observa en el Gráfico $\mathrm{N}^{\circ} 6$ a partir de la representación de los coeficientes lineales de regresión de estas variables. En general, los individuos con nivel educativo bajo tienen mayor probabilidad de experimentar una transición hacia el empleo que aquellos más educados. Por ejemplo, un desempleado promedio con educación primaria tiene una probabilidad de salir del desempleo $24 \%$ mayor que uno con educación universitaria. La mayor probabilidad de salir del desempleo que tienen los individuos de menor nivel educativo seguramente está relacionada con el salario de reserva más elevado que suelen tener aquellos con más años de educación y la mayor selectividad en la selección de empleos que ello le permite.

La dependencia de la duración, captada por las variables binarias construidas para los diferentes intervalos de duración, es estadísticamente significativa y tiene la forma no monotónica, similar a la de trabajos empíricos para otros países. ${ }^{21}$ La probabilidad de salir del desempleo crece durante los primeros meses de desempleo y luego decrece progresivamente, tal como muestra la representación de los coeficientes de regresión de estas variables (Gráfico $\mathrm{N}^{\circ} 7$ ).

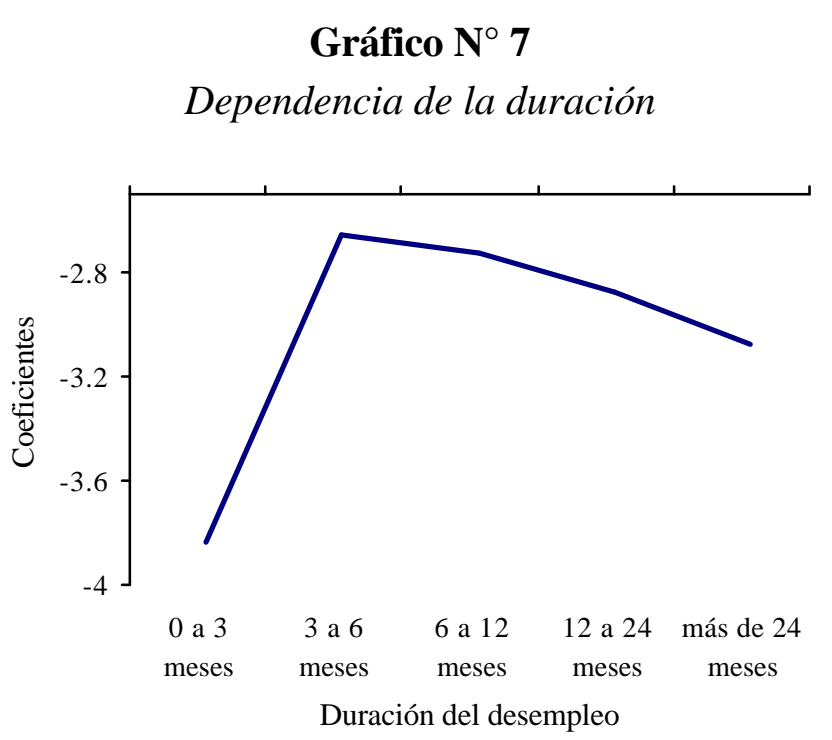

Entre el tercer y el sexto mes de desempleo, la probabilidad de experimentar un tránsito hacia el empleo para un individuo promedio es algo más de tres veces mayor que durante 
los primeros 3 meses. A su vez, para episodios de desempleo de más de 24 meses la probabilidad de salir del desempleo es 33,6\% menor que para aquellos que se encuentran entre el tercer y sexto mes. La reducción progresiva de la probabilidad de salir del desempleo para episodios de más de 3 meses podría estar asociada a la obsolescencia progresiva de las capacidades laborales del individuo conforme se incrementa el tiempo que éste ha estado sin empleo.

\section{La incidencia del ciclo económico}

El ciclo económico tiene una incidencia fuertemente significativa sobre la duración del desempleo. Todas las alternativas utilizadas para captar su efecto son consistentes entre sí e indican que durante las expansiones la probabilidad de experimentar un tránsito hacia el empleo es mayor que durante las recesiones. Desde el punto de vista teórico, esta relación no es unívoca. Ocurre que en los períodos de expansión económica tienden a incrementarse las oportunidades laborales, lo cual incide positivamente sobre la probabilidad de encontrar un empleo, pero también tienden a incrementarse las exigencias del trabajador para aceptar las oportunidades laborales que encuentra (aumenta su "salario de reserva"), reduciendo las posibilidades de que el trabajador acepte el empleo que encuentra. Un razonamiento similar puede realizarse para las fases descendentes del ciclo.

En las cinco especificaciones presentadas en la Tabla $\mathrm{N}^{\circ} 2$ se incorporan de la misma manera las características socioeconómicas y la dependencia de la duración, con los resultados comentados anteriormente. El primer intento de medir el efecto del ciclo se realizó incluyendo en el modelo variables dicotómicas anuales. Se observa que, en general, durante los períodos en los que la economía crece más la probabilidad de salir del desempleo es mayor, excepto durante el año 2002. Esto es lo que muestra el Gráfico $\mathrm{N}^{\circ} 8$ a partir de la representación conjunta del PBI desestacionalizado y de los coeficientes de regresión de las variables binarias anuales.

\footnotetext{
${ }^{21}$ Ver por ejemplo Bover, Arellano y Bentolila (1996) para un análisis del caso español.
} 


\section{Gráfico $\mathbf{N}^{\circ} 8$}

Duración del desempleo y ciclo económico

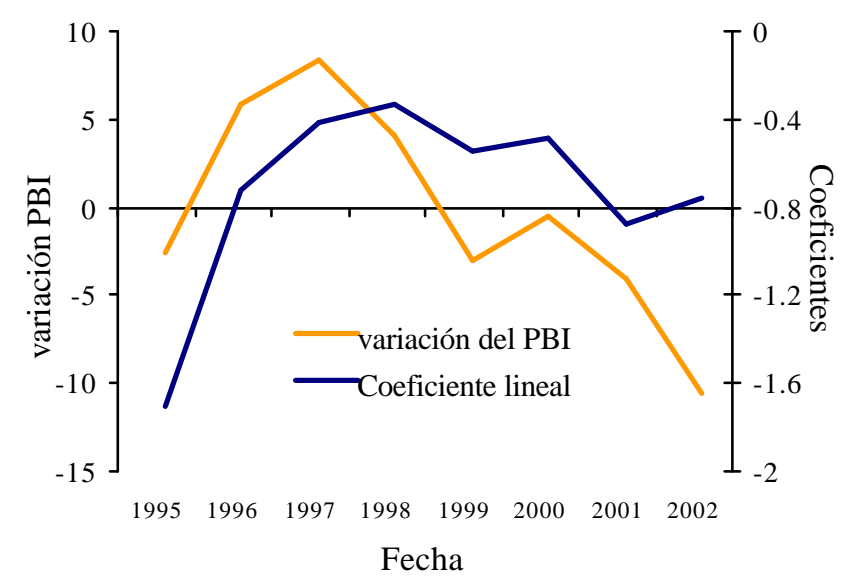

La probabilidad de salir del desempleo en 1997, año en el que la economía argentina creció algo más de $8 \%$, fue 3,5 veces más alta que en 1995, período en el cual la crisis financiera mejicana afectó negativamente el nivel de actividad económica del país. El comportamiento diferencial observado durante 2002 seguramente está relacionado con la puesta en marcha del plan Jefes y Jefas de Hogar Desocupados, ${ }^{22}$ que en la práctica funciona como un plan de empleo y que, al considerar empleados a los individuos que tienen un plan, eleva la probabilidad de experimentar una transición del desempleo hacia el empleo.

En el segundo modelo se capta el efecto del ciclo introduciendo la variación interanual del PBI trimestral como variable explicativa. Ante la evidencia recogida en el modelo anterior respecto a la relevancia del plan Jefes y Jefas de Hogar Desocupados para explicar la duración del desempleo, también se incorpora una variable dicotómica que intenta captar su efecto. Ambas resultaron estadísticamente significativas y presentaron los signos esperados. La probabilidad de salir del desempleo para un individuo promedio que se encuentra entre el tercer y el sexto mes de desempleo es $16,2 \%$ más elevada cuando el producto crece $4 \%$ que cuando cae a igual tasa. A su vez, durante el período de vigencia del plan social mencionado, la probabilidad de experimentar una transición hacia el empleo se incrementó $22,5 \%$ para el mismo individuo.

\footnotetext{
${ }^{22}$ Programa social que entrega \$ 150 a cambio de una contraprestación laboral. A los individuos que tienen este plan se los considera empleados.
} 
El ciclo, medido como la diferencia entre la serie de PBI y su tendencia, también resultó significativo como variable explicativa de la probabilidad de salir del desempleo. Cuando la economía se encuentra en la fase expansiva del ciclo esta probabilidad se incrementa. En el cuarto modelo se separan los efectos de las fases ascendente y descendente del ciclo y se observa que el impacto de las expansiones sobre la probabilidad de experimentar una transición hacia el empleo tiene una magnitud mayor que el de las recesiones. Dicha probabilidad es 4,8\% más elevada en la primera situación respecto a la segunda.

Finalmente, la tasa de desempleo del sector en el cual el individuo estuvo empleado la última vez que trabajó es en general significativa para explicar la probabilidad de salir del desempleo. A mayor tasa de desempleo, hecho que en el período que abarca la muestra está asociado a un menor nivel de actividad, dicha probabilidad se reduce. A su vez, existen diferencias notorias entre sectores productivos. La probabilidad de experimentar un tránsito hacia el empleo para un individuo promedio perteneciente al sector de la construcción es $30,9 \%$ superior a la de uno del sector industrial y 71,4\% mayor a la de uno del sector servicios. Para aquellos pertenecientes al sector industrial esta probabilidad es $31 \%$ mayor que para un integrante del sector servicios. ${ }^{23}$ El hecho de que la variable para la tasa de desempleo del sector primario no sea significativa seguramente está asociado a la escasa representatividad que tiene el sector primario en la EPH, dado que esta encuesta releva aglomerados urbanos.

\footnotetext{
${ }^{23}$ En todos los casos los cálculos se realizaron para una tasa de desempleo de $10 \%$.
} 


\section{Consideraciones finales}

Este trabajo presenta un conjunto de medidas alternativas de la incidencia del ciclo económico sobre la probabilidad de experimentar una transición del desempleo al empleo en Argentina. El resultado que se obtuvo fue que durante los períodos de recesión económica la probabilidad de salir del desempleo se reduce, tanto más cuanto más pronunciado sea el proceso contractivo. Este comportamiento no es homogéneo entre sectores productivos, incidiendo con mayor fuerza en el sector servicios.

El análisis contenido en este trabajo puede ser refinado y extendido. En primer lugar, podría ser interesante determinar cómo se modifican los resultados si no se considera empleados a los individuos que tienen un plan Jefes y Jefas de Hogar Desocupados dado que, aunque se trata de un plan de empleo, tiene características asistenciales más que de reinserción laboral. En segundo lugar, sería interesante explorar los efectos interacción entre la duración del desempleo y las variables incluidas en las regresiones, fundamentalmente sobre aquellas relacionadas con el ciclo económico. 


\section{Referencias Bibliográficas}

Arranz, J., Cid, J. y Muro, J. (2000). "La Duración del Desempleo en Presencia de Altas Tasas de Paro: el Caso de la Argentina". Asociación Argentina de Econo mía Política (AAEP). Documento de Trabajo Nro 1465.

Arrufat, J., Días Caferrata, A., Figueras, A. and Utrera, G. (1999). "Hysteresis and Structural Breaks in Regional Unemployment. Argentina 1980-1998". Asociación Argentina de Economía Política (AAEP). Documento de Trabajo Nro 1457.

Atkinson y Micklewright (1991) "Unemployment compensation and labor market transitions: acritical review”. Journal of Economic Literature, 29, págs. 1679-1727.

Bover, O., Arellano, M. y Bentolila, S. (1996). "Duración del Desempleo, Duración de las Prestaciones y Ciclo Económico”. Banco de España. Estudios Económicos Nro 57.

Díaz Cafferata, A.M., Figueras, A.J., Capmourteres, E.E. y Moncarz, P.E. (1997). "Indicadores de la Fuerza de trabajo. Un Análisis Regional: Argentina 1980-1996". Asociación Argentina de Economía Política (AAEP). Documento de Trabajo Nro 1241.

Féliz, M., Panigo, D. y Pérez, P. (2000). "Determinantes de la Desocupación en el Ambito Regional y su Influencia sobre la Implementación de Políticas de Desempleo". Asociación Argentina de Economía Política (AAEP). Documento de Trabajo Nro 1493.

Galiani, S. and Hopenhayn, H. (2001). "Duration and Risk of Unemployment in Argentina". Universidad de San Andrés (UDESA). Working Paper Nro 40.

Gasparini, L. Y otros (2002). "Características demográficas y pobreza en la Argentina". Banco Interamericano de Desarrollo (BID).

Heymann, D. (2000). "Políticas de Reforma y Comportamiento Macroeconómico: la Argentina en los Noventa". Comisión Económica para América Latina y el Caribe (CEPAL). Serie Reformas Económicas Nro 61.

Hopenhayn, H. (2001). "Labor Market Policies and Employment Duration: the Effects of Labor Market Reform in Argentina". Inter-American Development Bank (IADB). Working Paper R-407. 
Jenkins, S. P. (2004). “Survival Analysis”. University of Essex. United Kingdom.

Kiefer, N. (1988). "Economic Duration Data and Hazard Funcions". Journal of Economic Literature. Vol. XXVI, pp. 646-679.

Lamarche, C., Porto, A. y Sosa Escudero, W. (1998). “Aspectos regionales del desempleo en Argentina". Asociación Argentina de Economía Política (AAEP). Documento de Trabajo Nro 1327.

Lancaster, T. (1990). “The Econometric Analysis of Transition Data”. Econometric Society Monograph $\mathrm{N}^{\circ}$ 17. Cambridge University Press.

Llach, J. y Llach, L. (1998). "Cancelando la Hipoteca. Hiperinflación, Reforma de la Economía. Empleo y Desempleo en la Argentina de los 90”. Inter-American Development Bank (IADB). Working Paper 385.

Pessino, C. (1996), “La anatomía del desempleo”. Desarrollo Económico. Número Especial. Volumen 36, pp. 223-266. 